\title{
SOUND PROPAGATION IN FLUIDS AND NEUTRON SPECTRA ${ }^{\text {is }}$
}

\author{
M.J. ZUILHOF \\ Instituut voor Theoretische Fysica, Rijksuniversiteit Utrecht, 3508 TA Utrecht, The Netherlands
}

E.G.D. COHEN

The Rockefeller University, 1230 York Avenue, New York, NY 10021, USA

and

I.M. DE SCHEPPER

Interuniversitair Reactor Instituut, $2629 \mathrm{JB}$ Delft, The Netherlands

Received 26 April 1984

\begin{abstract}
Using a model kinetic equation the properties of very short wavelength sound modes in fluids are studied over a wide range of wavenumbers and densities. The main features, in particular propagation gaps at high densities, agree with those found in real fluids and molecular dynamics simulations.
\end{abstract}

Using a kinetic theory for hard sphere fluids, it has been predicted [1] that the dynamic structure factor $S(k, \omega)$ can be described as a function of frequency $\omega$ in terms of three extended hydrodynamic modes, a heat and two sound modes, for wavenumbers $k$ far exceeding the hydrodynamic regime, i.e. for $k \sigma \gg 1$, where $\sigma$ is the diameter of the hard spheres and $k \sigma \leqq 1$ is representative of the hydrodynamic region [1]. Such a description has been validated by neutron scattering experiments on liquid argon at a number of pressures [2] as well as by molecular dynamics (MD) calculations on dense hard sphere [3] and Lennard-Joneslike fluids [4] up to $k \sigma \approx 15$. A more detailed discussion of the extended heat mode and in particular its relevance for the width and height of neutron spectra was given recently [5]. Here we present new results for the extended sound modes together with results for those kinetic modes that are needed to understand the rather complicated behavior of the sound modes as a function of the number density $n$.

The starting point is an approximate linear kinetic operator $L(k)$, that determines the time evolution of

\footnotetext{
Work supported by I.R.I. Delft.
}

small disturbances from equilibrium as well as all the equilibrium time correlation functions of a hard sphere fluid. This, so-called, generalized Enskog operator $L(k)$ is the sum of a free streaming, a collision and a mean field term

$$
L(k)=-\mathrm{i} k \cdot \boldsymbol{v}+n g(\sigma) \Lambda_{k}+n A_{k} .
$$

Here $\boldsymbol{k}$ is a wave vector, $\boldsymbol{v}$ the velocity, $g(\sigma)$ the radial distribution function of two hard spheres at contact; $\Lambda_{k}$ a binary collision term, while $A_{k}$ contains the static structure factor $S(k)$. For explicit expressions of the operators $\Lambda_{k}$ and $A_{k}$, which act on functions of $\boldsymbol{v}$, we refer to elsewhere [1,5]. $L(k)$ is applied through a spectral decomposition in eigenmodes,

$L(k)=\sum_{j}\left|\Psi_{j}(k, v)\right\rangle z_{j}(k)\left\langle\Phi_{j}(k, v)\right|$,

where the $z_{j}(k)$ are in general complex eigenvalues of $L(k)$ and $\Psi_{j}$ and $\Phi_{j}$ denote the corresponding right and left eigenfunctions respectively. The bracket notation in eq. (2) refers to the inner product

$\langle f \mid h\rangle=\left\langle f^{*} h\right\rangle=\int \mathrm{d} \boldsymbol{v} \varphi(v) f^{*}(\mathbf{v}) h(\mathbf{v})$, 
where the equilibrium distribution function $\varphi(v)=$ $(\beta M / 2 \pi)^{3 / 2} \exp \left(-\beta M v^{2} / 2\right)$, with $M$ the mass of a hard sphere, $\beta=\left(k_{\mathrm{B}} T\right)^{-1}$, where $k_{\mathrm{B}}$ is the Boltzmann constant and $T$ the temperature. As pointed out before, $S(k, \omega)$ can be expressed directly in terms of $L(k)$ or in its spectral decomposition as an infinite sum of lorentzians

$$
\begin{gathered}
S(k, \omega)=\pi^{-1} S(k) \operatorname{Re}\left\langle[\mathrm{i} \omega-L(k)]^{-1}\right\rangle \\
\quad=\pi^{-1} S(k) \sum_{j} \frac{A_{j}(k)}{i \omega-z_{j}(k)},
\end{gathered}
$$

where

$$
A_{j}(k)=\left\langle\Psi_{j}(k, v)\right\rangle\left\langle\Phi_{j}^{*}(k, v)\right\rangle .
$$

The $A_{j}$ and $z_{j}(j= \pm$ ) for the sound modes appear in complex conjugate pairs for almost all $k$ values.

For the following it is relevant to remark that (a) the collision term $n g(\sigma) \Lambda_{k}$ in eq. (1) is of order $t_{\mathrm{E}}^{-1}$ for all $k$, depends on $k$ only through the parameter $k \sigma$ and approaches for $k \sigma>2 \pi$ the limit $n g(\sigma) \Lambda_{\infty}$, (b) the importance of the $-\mathbf{i} k \cdot v$ and $n A_{k}$ terms in eq. (1) relative to the collision term is given by the parameter $k l_{\mathrm{E}}$ and (c) $n A_{k}$ is in addition characterized by the strength of the mean field $1-1 / S(k)$, which varies on the scale $k \sigma$ and approaches zero when $k \sigma$ $>2 \pi$ for all densities. Here $t_{\mathrm{E}}$ and $l_{\mathrm{E}}$ are the mean free time and path respectively with $\sigma / l_{\mathrm{E}}=(\pi / 2)^{1 / 2} \times$ $t_{\sigma} / t_{\mathrm{E}}=\sqrt{2} \pi n \sigma^{3} g(\sigma)$ and $t_{\sigma}=(\beta M)^{1 / 2} \sigma / 2$. In addition to $L(k)$ we have to consider the operator [1] $L^{\mathrm{s}}(k)=-\mathrm{i} \boldsymbol{k} \cdot \boldsymbol{v}+n g(\sigma) \Lambda_{\infty}$, which governs the time evolution of the self-correlation functions in the fluid. For, $L(k)$ tends to $L^{\mathrm{s}}(k)$ for $k \sigma>2 \pi$, since then $\Lambda_{k} \rightarrow \Lambda_{\infty}$ and $A_{k} \rightarrow A_{\infty}=0$. Therefore each eigenmode of $L(k)$ approaches an eigenmode of $L^{\mathrm{s}}(k)$ and in particular each $z_{j}(k)$ tends to one of the eigenvalues $z_{j}^{\mathrm{s}}(k)$ of $L^{\mathrm{s}}(k)$ for $k \sigma>2 \pi$. For $k \rightarrow 0, A_{k} \rightarrow 0$ and the eigenvalues of $L(k)$ and $L^{\mathrm{s}}(k)$ tend to $n g(\sigma)$ times those of the Boltzmann collision operator $\Lambda_{0}$ and the collision operator of the Rayleigh model [6] $\Lambda_{\infty}$ respectively, for both of which all eigenvalues are real, $\leqslant 0$ and independent of the density, so that $z_{j}(0)$ and $z_{j}^{\mathrm{s}}(0)$ depend on the density through $n g(\sigma)$, i.e. $t_{\mathrm{E}}^{-1}$.

The eigenmodes of $L(k)$ and $L^{\mathrm{s}}(k)$ are determined by approximating $\Lambda_{k}$ in a Bhatnagar-Cross - Krooklike fashion using 11 of a complete $\operatorname{set}\left\{\varphi_{j}\right\}$ of orthonormal polynomia in $\boldsymbol{v}$, that can be labeled with the "quantum numbers" $j=(r, l, m)$. These polynomia are in general given by $N_{r, l} v^{l} L_{r}^{(l+1 / 2)}\left(\beta M v^{2} / 2\right) Y_{l}^{(m)}$ $(v / v)$, where the $L_{r}^{(l+1 / 2)}$ are associated Laguerre polynomia with normalization constants $N_{r, l}$ and the $Y_{l}^{(m)}$ are spherical harmonics with the $z$-axis taken in the $k$-direction. For the eigenmodes of $L(k)$ and $L^{\mathrm{s}}(k)$, $m$ is an exact quantum number that vanishes for the heat and sound modes. The 11 polynomials used to represent $\Lambda_{k}$ are given elsewhere, as is the method $[1,5]$ to determine the six lowest eigenmodes of $L(k)$ and $L^{\mathrm{s}}(\boldsymbol{k})$ with $m=0$. For all $k$, these eigenmodes of $L(k)$ and $L^{\mathrm{s}}(k)$ can be divided into (extended) hydrodynamic modes, with eigenvalues that go to zero for $k \rightarrow 0$ and kinetic modes with eigenvalues that approach non-zero negative values for $k \rightarrow 0$. For $L^{\mathrm{s}}(\boldsymbol{k})$, these are the self-diffusion mode $(j=\mathrm{D})$ and five kinetic modes, while for $L(k)$ they are the heat $(j=\mathrm{h})$, two sound $(j= \pm)$ and three kinetic modes, one of which is real and is connected with the heat mode, while the other two are complex conjugate for almost all $k$ values and are connected with the sound modes. Results have been obtained for $k$ up to $k \sigma=60$ and reduced densities $V_{0} / V=n \sigma^{3} / \sqrt{2}$ from 0.1 to 0.7 where $V_{0}$ is the volume of close packing of the hard sphere fluid.

We find in our calculation that of the 11 orthonormal polynomia $\varphi_{j}$, those most important for the lowest six eigenmodes of $L(k)$ and $L^{\mathrm{S}}(\boldsymbol{k})$ with $m=0$ have quantum numbers $(r, l)$ equal to $(0,0) ;(0,1) ;(1,0)$; $(1,1) ;(2,0)$ and $(0,2)$ for $j=1, \ldots, 6$ respectively and are given by $\varphi_{1}=1, \varphi_{2}=(\beta M)^{1 / 2} v_{z}, \varphi_{3}=\left(\beta M v^{2}-3\right) /$ $\sqrt{6}, \varphi_{4}=(\beta M / 10)^{1 / 2} v_{z}\left(\beta M v^{2}-5\right), \varphi_{5}=$ $\sqrt{30}\left[\left(\beta M v^{2}\right)^{2}-10 \beta M v^{2}+15\right] / 60$ and $\varphi_{6}=$ $\sqrt{3} \beta M\left(v^{2}-3 v_{z}^{2}\right) / 6$. In this connection, we note that for $k=0, \varphi_{1}, \ldots, \varphi_{6}$ include the exact zero eigenfunctions of $L(0)(j=1,2,3)$ and $L^{\mathrm{s}}(0)(j=1)$. Since in addition to $m$ also $l$ is a good quantum number for $L(0)$ and $L^{\mathrm{s}}(0)$, the polynomia $\varphi_{j}$ with $j=4,5,6$ and those with $j=2, \ldots, 6$ represent very good approximations to the lowest kinetic eigenfunctions of $L(0)$ and $L^{\mathrm{s}}(0)$ respectively. Although for $k \neq 0, l$ is no longer a good quantum number, the $\varphi_{j}$ with $j=1, \ldots, 6$ are still dominant in the six lowest eigenmodes of $L(k)$ and $L^{\mathrm{s}}(k)$ as long as $k l_{\mathrm{E}}<1$. For larger values of $k$, $\varphi_{j}$ with $j>6$ are increasingly needed for a satisfactory representation, however we shall still continue to use $j=1, \ldots, 6$ as labels of the eigenmodes.

We first discuss the results for $L^{\mathrm{s}}(k)$. The reduced eigenvalues $z_{j}^{\mathrm{S}}(k) t_{\mathrm{E}}$ as functions of $k l_{\mathrm{E}}$ are indepen- 


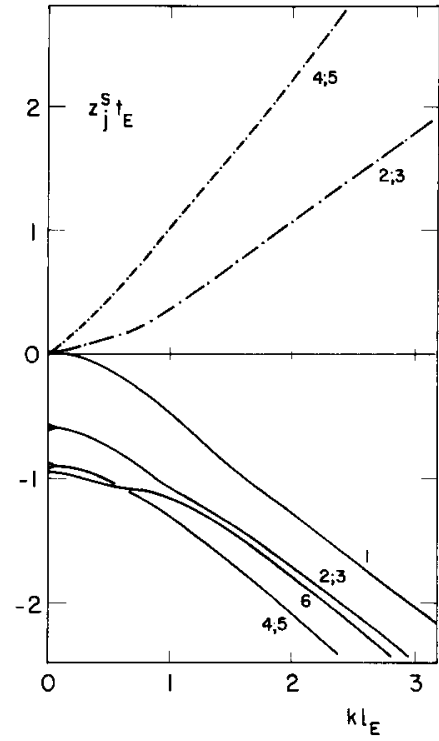

Fig. 1. Reduced six lowest eigenvalues $z_{j}^{\mathrm{s}}(k) t_{\mathrm{E}}$ of $L^{\mathrm{s}}(k)$ as functions of $k l_{\mathrm{E}}$. Positive values refer to absolute values of imaginary parts and negative values to real parts. The diffusion mode eigenvalue $z_{1}(k)$ and $z_{6}(k)$ are real for all $k$. dent of the density and shown in fig. 1, using the labeling described above. For $k=0$, the $z_{j}^{\varsigma}(0) t_{\mathrm{E}}$ are: 0 ; $-0.596 ;-0.624 ;-0.899 ;-0.944$ and -0.961 for $j=$ $1, \ldots 6$ respectively. The eigenvalues $z_{1}^{\mathrm{s}}(k)=z_{\mathrm{D}}^{\mathrm{s}}(k)$ and $z_{6}^{\mathrm{s}}(k)$ are real up to $k l_{\mathrm{E}}=10$ at least. However, $z_{2}^{\mathrm{s}}(k)$ and $z_{3}^{\mathrm{s}}(k)$ are almost degenerate and real only up to $k l_{\mathrm{E}}=0.055$; for larger $k$ they become complex conjugate, with the corresponding eigenfunctions approximately given by $\varphi_{2} \pm \varphi_{3}$, at least for $k l_{\mathrm{E}}<1$. Similarly $z_{4}^{\mathrm{s}}(k)$ and $z_{5}^{\mathrm{s}}(k)$ are almost degenerate, real only up to $k l_{\mathrm{E}}=0.032$ and complex conjugate for larger $k$, with corresponding eigenfunctions approximately given by $\varphi_{4} \pm \varphi_{5}$ for $k l_{\mathrm{E}}<1$. In general, for $k l_{\mathrm{E}}>1$, the $z_{j}^{s}(k)$ show a linear behavior as a function of $k$.

We next discuss the results for the six lowest eigenmodes of $L(k)$, which for large $k$, approach corresponding modes of $L^{\mathrm{s}}(k)$. The reduced eigenvalues $z_{j}(k) t_{\sigma}$ as functions of $k \sigma$ are shown in fig. 2 for various reduced densities $V_{0} / V$. In order to facilitate the conversion of $k \sigma$ into $k l_{\mathrm{E}}$ we place arrows in our figures that point to that value of $k \sigma$ where $k l_{\mathrm{E}}=1$ (cf. fig. 2). For $k=0$ and all densities the lowest eigenvalue of
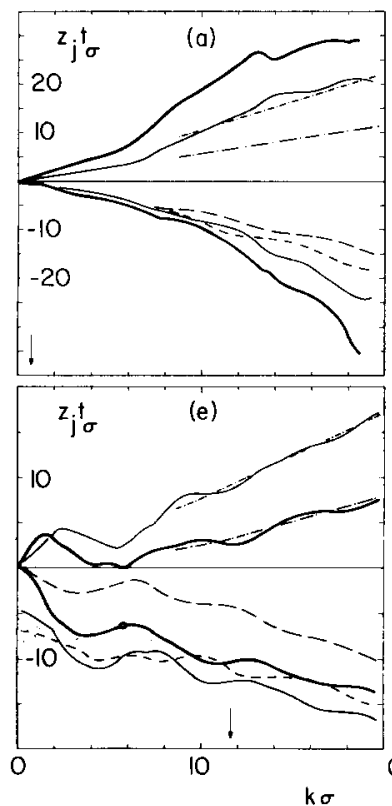

$k \sigma$
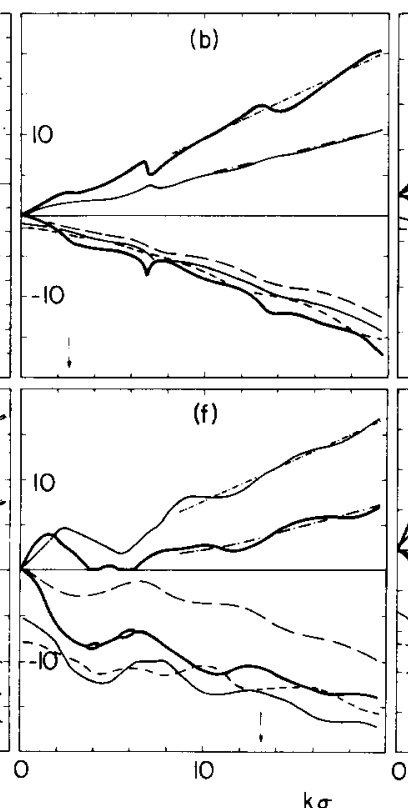

$k \sigma$
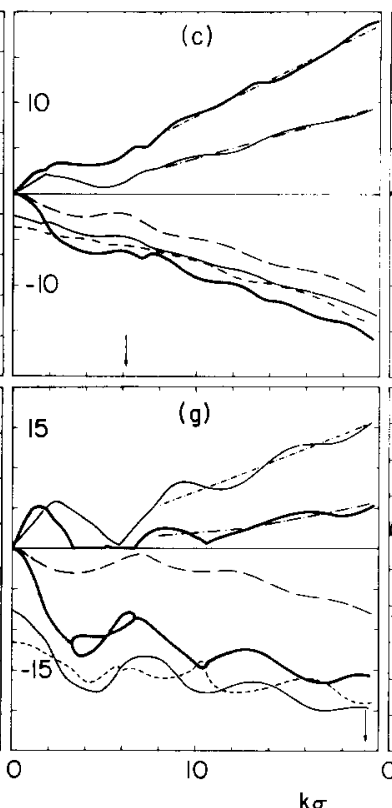

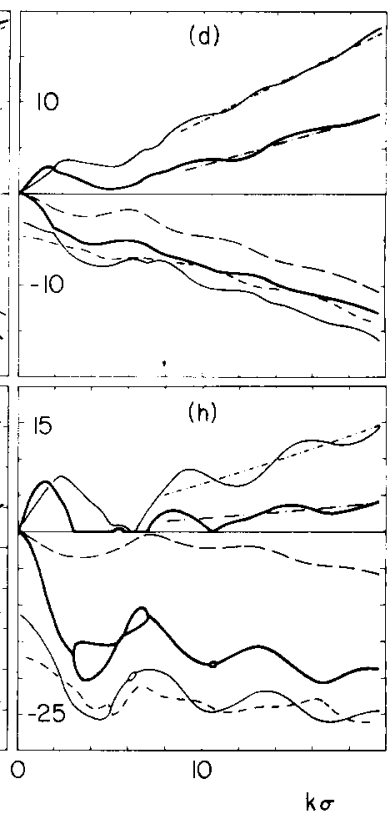

Fig. 2. Reduced six lowest eigenvalues $z_{j}(k) t_{\sigma}$ of $L(k)$ as function of $k_{\sigma}$ for reduced densities $V_{0} / V=0.10(\mathrm{a}) ; 0.25(\mathrm{~b}) ; 0.40(\mathrm{c})$; $0.45(\mathrm{~d}) ; 0.525(\mathrm{e}) ; 0.55(\mathrm{f}) ; 0.625(\mathrm{~g})$ and $0.70(\mathrm{~h})$. Positive values refer to absolute values of imaginary parts and negative values to real parts of the sound $(j=2,3 ;$ fat solid line) and kinetic sound $(j=4,5 ;$ solid line) mode eigenvalues. The heat $(j=1 ;--)$ and kinetic heat $(j=6 ;-\ldots)$ mode eigenvalues are real for all $k$. The arrows point to where $k l \mathrm{E}=1$. The dash-dotted curves refer to imaginary parts of the eigenvalues of $L^{\mathrm{s}}(k)$ (cf. fig. 1). 
$L(0), z_{j}(0)=0$ and is degenerate for $j=1,2,3$, while $z_{j}(0) t_{\mathrm{E}}=-0.494 ;-0.505 ;-0.750$ for $j=4,5,6$. For small increasing $k \neq 0$ and all densities, the three eigenmodes that develop from the threefold degenerate zero eigenvalue are the usual three hydrodynamic modes: the heat mode with eigenvalue $z_{\mathrm{h}}(k)=-\alpha_{\mathrm{E}} k^{2}$ and the two sound modes with eigenvalues $z_{ \pm}(k)= \pm \mathrm{i} c k-$ $\Gamma_{\mathrm{E}} k^{2}$, where $\alpha_{\mathrm{E}}$ and $\Gamma_{\mathrm{E}}$ are the Enskog values of the heat and sound damping coefficients respectively and $c$ is the adiabatic sound velocity. For later we remark that $z_{\mathrm{h}}$ and $z_{ \pm}$are continuous functions of the density. To these three eigenvalues correspond three eigenfunctions that are linear combinations of $\varphi_{1}, \varphi_{2}$ and $\varphi_{3}$, where $\varphi_{1}$ dominates the heat mode, while $\varphi_{2}$ and $\varphi_{3}$ dominate the two sound modes [1]. We shall label for all $k$ the extended heat mode - i.e. the hydrodynamic heat mode and its continuous extension to larger values of $k$ - by 1 and correspondingly the two extended sound modes by 2,3 . For all densities the eigenvalues $z_{4}(k)$ and $z_{5}(k)$, corresponding to what we shall call "kinetic sound modes", are almost degenerate, become complex when $k \sigma$ exceeds approximately 0.01 , after which they are each others' complex conjugate. $z_{1}(k)$ and the eigenvalue $z_{4}(k)$ of the "kinetic heat mode", remain real for all $k$-values (cf. fig. 2) and approach $z_{1}^{\mathrm{s}}(k)$ and $z_{4}^{\mathrm{s}}(k)$ respectively.

The approach of the pairs of complex sound mode eigenvalues $z_{2}, z_{3}$ and $z_{4}, z_{5}$ for large $k$ to pairs of $z^{s}$ eigenvalues depends in a complicated way on the density. For sufficiently large densities, $V_{0} / V \geqslant 0.43$, the pairs of eigenvalues $z_{2}, z_{3}$ and $z_{4}, z_{5}$ approach the pairs $z_{2}^{\mathrm{s}}, z_{3}^{\mathrm{s}}$ and $z_{4}^{\mathrm{s}}, z_{5}^{\mathrm{s}}$ respectively (cf. figs. $2 \mathrm{~d}-\mathrm{h}$ ). For smaller densities $0.25 \leqslant V_{0} / V<0.43$, the pair $z_{2}, z_{3}$ approaches $z_{4}^{\mathrm{s}}, z_{5}^{\mathrm{s}}$, while the pair $z_{4}, z_{5}$ approaches $z_{2}^{\mathrm{s}}, z_{3}^{\mathrm{s}}$ (cf. figs $2 \mathrm{~b}, \mathrm{c}$ ). For still lower densities, the general behavior is not clear, but at $V_{0} / V=$ 0.1 , for instance, the pair $z_{2}, z_{3}$ approaches a pair of kinetic modes of $L^{\mathrm{s}}(k)$ even higher than $z_{4}^{\mathrm{s}}, z_{5}^{\mathrm{s}}$, while the pair $z_{4}, z_{5}$ again approaches $z_{4}^{\mathrm{s}}, z_{5}^{\mathrm{s}}$ (cf. fig. 2a). As a result, although for small fixed $k$, the sound mode eigenvalues $z_{2}, z_{3}$ are continuous functions of the density, for $k \sigma \geqslant 1.67$ they jump discontinously from values near one $z^{s}$-pair to those near another $z^{s}$. pair (compare e.g. fig. $2 \mathrm{c}$ with fig. $2 \mathrm{~d}$ ) as a function of the density. For $V_{0} / V<0.43$, the $\left|\operatorname{Im} z_{j}\right|$ of the kinetic sound modes $j=4,5$ lie below that of the (normal) sound modes $j=2,3$ and exhibit a minimum at $k \sigma \approx 6$ (cf. fig. 2). Related to this complicated be- havior of the sound modes for large $k$, there is also an interesting behavior as a function of density at intermediate values of $k$. In order to discuss this, we introduce the sound frequency $\omega_{\mathrm{s}}(k)=\left|\operatorname{Im} z_{2,3}(k)\right|$ and the sound damping $z_{\mathrm{s}}(k)=\operatorname{Re} z_{2,3}(k)$. While for all densities and small $k \sigma \lesssim 1$, a hydrodynamic behavior with $\omega_{\mathrm{s}}(k)=c k$ and $z_{\mathrm{s}}(k)=-\Gamma_{\mathrm{E}} k^{2}$ obtains, for $k \sigma>1$ $\omega_{s}(k)$ and $z_{s}(k)$ start to oscillate around the imaginary and real parts of those eigenvalues of $L^{\mathrm{s}}(k)$ to which they approach (cf. figs. 1,2).

For $V_{0} / V \lesssim 0.52$ the sound modes are always propagating, i.e. $\omega_{\mathrm{s}}(k)>0$ for all $k$, exhibiting a distinct Landau-like minimum around $k \sigma \approx 6$ (cf. fig. $2 \mathrm{~d}$ ) that decreases with decreasing density. However, for $0.52 \leqslant$ $V_{0} / V \leqslant 0.54$ the sound modes show a propagation gap around $k \sigma \approx 6$ i.e. a region where $\omega_{\mathrm{s}}(k)=0$ and the modes do not propagate. In such a region there are two purely damped modes with different damping coefficients $\operatorname{Re} z_{2}(k) \neq \operatorname{Re} z_{3}(k)$ (cf. fig. $2 \mathrm{e}$ ). For $0.54 \leqslant V_{0} / V \leqslant 0.69$ there are two gaps around $k \sigma \approx 6$ separated by a small intermediate $k$-region where the modes propagate again (cf. fig. 2f, g), while for 0.69 $\leqslant V_{0} / V \leqslant 0.70$ a third gap appears at $k \sigma \approx 11$ (cf. fig. $2 \mathrm{~h}$ ). We note that at $V_{0} / V=0.7$ also the kinetic sound modes $z_{4}, z_{6}$ exhibit a propagation gap at $k \sigma \approx 6$ and that propagation gaps persist to the highest densities considered. Thus except at $k \sigma \leqslant 1$, in the hydrodynamic region, the behavior of the sound modes as a function of $k$ is qualitatively quite different for various density regimes. In addition, sharp irregularities in $z_{2,3}(k)$ appear when they are very close to $z_{4,5}(k)$, e.g. at $k \sigma=1.67$ in figs. $2 \mathrm{c}$ and d, to $z_{6}(k)$, e.g. at $k \sigma=$ $10.5 \mathrm{in} \mathrm{fig.} 2 \mathrm{~g}$ or to even higher modes, e.g. at $k \sigma=6.9$ in fig. $2 b$.

The appearance of a propagation gap at high densities and around $k \sigma \approx 6$ is due to the decreasing importance of the free streaming term $-\mathrm{i} k \cdot \boldsymbol{v}$ relative to the other two terms on the right-hand side of eq. (1) for increasing densities. This can qualitatively be understood as follows. We first note that the eigenmodes of $L(k)$ are also eigenmodes of the infinite matrix $L_{j, l}(k) \equiv\left\langle\varphi_{j}^{*} L(k) \varphi_{l}\right\rangle$. The simplest approximation to $L_{j, l}$ which still contains one heat and two sound modes is the matrix $L_{j, l}$ with $1 \leqslant j, l \leqslant 3$. Using eq. (1) and ref. [1] one easily finds that $L_{1,1}(k)=L_{1,3}(k)=$ $L_{3,1}(k)=0, L_{2,1}(k)=-\mathrm{i} k l_{\mathrm{E}} t_{\mathrm{E}}^{-1}(\pi / 8)^{1 / 2}, L_{1,2}(k)=$ $L_{2,1}(k) / S(k), L_{2,2}(k)=-d_{2}(k) t_{\mathrm{E}}^{-1}, L_{3,3}(k)=$ $-d_{3}(k) t_{\mathrm{E}}^{-1}$ and $L_{2,3}(k)=L_{3,2}(k)=-\mathrm{i} f(k) t_{\mathrm{E}}^{-1}$, with 
$d_{2}(k)=2\left[1-j_{0}(k \sigma)+2 j_{2}(k \sigma)\right] / 3, d_{3}(k)=2[1-$ $\left.j_{0}(k \sigma)\right] / 3$ and $f(k)=(\pi / 12)^{1 / 2}\left[k l_{\mathrm{E}}+\sqrt{2} j_{1}(k \sigma)\right]$. The factor $k l_{\mathrm{E}} / S(k)$ in $L_{1,2}(k)$ is due to the terms $-\mathrm{i} k \cdot$ and $n A_{k}$ in $L(k)$, the factors $k l_{\mathrm{E}}$ in all remaining $L_{j, l}$ are due to the free streaming term $-\mathrm{i} k \cdot \mathbf{v}$ alone and the constant terms and the spherical Bessel functions $j_{l}(k \sigma)$ are due to $n g(\sigma) \Lambda_{k}$ in $L(k)$. We then find that the eigenmodes of $L_{j, l}$ with $j, l \leqslant 3$ approximate well the heat and sound modes of the full kinetic operator $L(k)$ as long as $k l_{\mathrm{E}}<1$, since the heat and sound mode eigenfunctions are dominated by $\varphi_{1}, \varphi_{2}$, $\varphi_{3}$ for $k l_{\mathrm{E}}<1$. Also, we find that indeed only for $V_{0} / V \geqslant 0.52$ propagation gaps in $\omega_{s}(k)$ appear. This is illustrated in fig. 3 for $V_{0} / V=0.70$ where one observes, in particular, the appearance of two gaps around $k \sigma \approx 6$, just as in fig. $2 \mathrm{~h}$.

Physically the appearance and disappearance of propagation gaps is due to a competition between elasticity and dissipation in the fluid. This can be seen already by an even further simplification of the model discussed above, i.e. by considering $L_{j, l}$ with $2 \leqslant j, l \leqslant 3$ only. Multiplying $\varphi_{2}$ and $\varphi_{3}$ with a nonequilibrium distribution function corresponding to a small disturbance from $\varphi(v)$ that spatially varies as $\exp (\mathrm{i} k \cdot r)$ and integrating over $\boldsymbol{v}$ gives for the normalized amplitudes $u(k, t)$ of the macroscopic velocity and $e(k, t)$ of the macroscopic energy the coupled equations

$$
\frac{\partial}{\partial t}\left(\begin{array}{c}
u(k, t) \\
e(k, t)
\end{array}\right)=\frac{1}{t_{\mathrm{E}}}\left(\begin{array}{cc}
-\mathrm{d}_{2}(k) & \mathrm{i} f(k) \\
\mathrm{i} f(k) & -d_{3}(k)
\end{array}\right)\left(\begin{array}{c}
u(k, t) \\
e(k, t)
\end{array}\right) .
$$

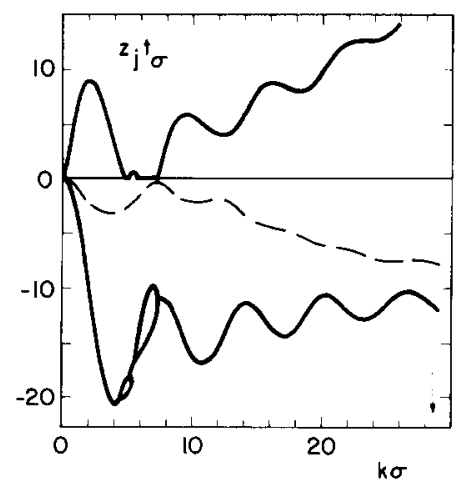

Fig. 3. Reduced eigenvalues $z_{j}(k) t_{\sigma}$ of the matrix $L_{j, l}$ with $1 \leqslant j, l \leqslant 3$ at $V_{0} / V=0.70$. Positive and negative values refer to imaginary and real parts respectively of the sound $(-)$ and heat $(--)$ mode eigenvalues. The arrow points to where $k l_{\mathrm{E}}=1$.
Clearly, while $d_{2,3}(k) \geqslant 0$ are damping (dissipative) terms, $f(k)$ is an elastic (restoring force) term. The character of the normal solutions of eq. (4), i.e. of the sound modes, is determined by the eigenvalues

$$
\begin{aligned}
& z_{2,3}(k) t_{\mathrm{E}} \\
& \quad=-\frac{1}{2}\left\{d_{2}+d_{3} \pm\left[\left(d_{2}-d_{3}\right)^{2}-(2 f)^{2}\right]^{1 / 2}\right\}
\end{aligned}
$$

of the matrix in eq. (4). Noting that $d_{2}-d_{3}=4 j_{2}(k \sigma) / 3$ while $f \sim k l_{\mathrm{E}}+\sqrt{2} j_{1}(k \sigma)$ one sees immediately that for $k \sigma \ll 1$, i.e. in the hydrodynamic regime, always propagation occurs since then $d_{2}-d_{3} \leqslant 2 f$ as $j_{l}(k \sigma)$ $\sim(k \sigma)^{l}$. For $k \sigma \gtrsim 1$ the character of $z_{2.3}$ is determined by the sign of $d_{2}-d_{3}-2 f$ or equivalently $h(k \sigma)-k l_{\mathrm{E}}$, where $h(k \sigma)=(32 / 9 \pi)^{1 / 2} j_{2}(k \sigma)-\sqrt{2} j_{1}(k \sigma)$. To determine this as a function of density we have plotted in fig. $4 h(k \sigma)$ and $k l_{\mathrm{E}}=\left(\sigma / l_{\mathrm{E}}\right)^{-1} k \sigma$ as functions of $k \sigma$. Then, when $h(k \sigma)<k l_{\mathrm{E}}$ or $\sigma / l_{\mathrm{E}}<16$ (i.e. $V_{0} / V$ $<0.59), d_{2}-d_{3}<2 f, z_{2,3}$ are complex and propagation of sound always occurs, while for $\sigma / l_{\mathrm{E}} \geqslant 16$ (i.e. $V_{0} / V \geqslant 0.59$ ) a propagation gap exists around the first maximum of $h(k \sigma)$ at $k \sigma=5$. The figure also illustrates that a second gap can appear around $k \sigma=11.6$ where $h(k \sigma)$ has its second maximum. Since, however, $h(k \sigma) \leqslant 0$ for $k \rightarrow 0$ and $h(k \sigma) \rightarrow 0$ for $k \rightarrow \infty$ propagation will prevail at small as well as at large $k$, so that the number of gaps is limited.

We note that, unlike in the case of the heat mode discussed in a previous article [5], the behavior of the sound modes derived from $L(k)$ (cf. fig. 2) appears to be very insensitive to the static structure factor $S(k)$. For, setting $S(k)=1$ in $L(k)$, hardly changes

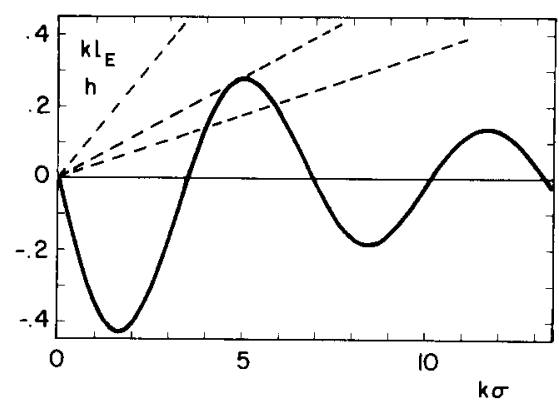

Fig. 4. The solid line represents $h(k \sigma)=(32 / 9 \pi)^{1 / 2} j_{2}(k \sigma)-$ $\sqrt{2} j_{1}(k \sigma)$ and the dashed lines $k l_{\mathrm{E}}$ at $V_{\mathrm{O}} / V=0.45,0.59$ and 0.70 , from top to bottom respectively, as functions of $k o$. For $k l_{\mathrm{E}}<h(\pi \sigma)$ gaps are present in the sound dispersion curves given by eq. (5). 
these sound dispersion curves. Furthermore, the approximation given by eq. (5) leads to similar dispersion curves, including propagation gaps, while $S(k)$ does not appear in this approximation at all. Also, the usual linearized Navier-Stokes equations, which do contain the competition between elastic and dissipative forces but not $S(k)$, exhibit for certain values of the thermodynamic and transport coefficients a sound dispersion curve with a propagation gap not unlike that sketched in fig. 3 [7]. These results would suggest that the absence of propagation gaps in the second dispersion curves of glasses [8] and regular solids [9] is due to the fact that, in general, dissipative forces are smaller and elastic forces are at least of the order of magnitude of those in dense fluids.

We conclude with a number of consequences of the behavior of the sound modes on the neutron spectra of fluids. (1) As discussed before [1] the existence of sound modes in a hard sphere fluid can not be deduced simply from the existence of visible (Brillouin-like) side peaks in $S(k, \omega)$. In fact we find that visible side peaks in $S(k, \omega)$ are absent for $k \sigma \gtrsim 0.5$ but that $S(k, \omega)$ is still well represented by one heat and two sound modes up to $k l_{\mathrm{E}} \sim 1$. For this region, i.e. $\frac{1}{2} \leqslant k \sigma \leqslant \sigma / l_{\mathrm{E}}$, which covers at high densities most of the neutron scattering region, i.e. $1 \lesssim k \sigma \lesssim 20$, a study of $\omega^{2} S(k, \omega)$ is more appropriate to reveal properties of the sound modes. For still larger values of $k$, i.e. $1 \leqq k l_{\mathrm{E}} \lesssim 2$ all the six modes (i.e. three extended hydrodynamic and three kinetic modes) discussed here are needed to describe $S(k, \omega)$ satisfactorily. (2) The propagation gap predicted for the hard sphere fluid has first been observed in real fluids like liquid $\mathrm{Ar}$ and liquid $\mathrm{Ne}$, by an analysis of $S(k, \omega)$ in terms of three Lorentz lines [cf. eq. (3)] [2]. In addition they are found in MD simulations of dense Lennard-Jones-like fluids [4]. (3) We note that for $V_{0} / V \approx 0.45$, a reduced density that corresponds to that of liquid helium at low temperatures, the behavior of the sound modes of a classical hard sphere gas and liquid He are very similar
[10]. For, both have a Landau-like sound dispersion curve, a nearby kinetic sound mode, which both have oscillating widths on the scale $k \sigma$, just like in fig. $2 \mathrm{~d}$. (4) One could wonder to what extent the appearence of a propagation gap is not only unique but also universal in dense fluids. To investigate this one might study whether a Landau-like dispersion curve (as in fig. 2d) would appear in liquid Ar at lower densities, or whether a propagation gap (as in fig. $2 \mathrm{~g}$ ) in the dispersion curve would appear in liquid helium at higher densities (and temperatures) than considered so far. (5) Since the propagation gap and the de Gennes minimum at high densities both occur near $k \sigma=6$, a sharpening in addition to a narrowing of $S(k, \omega)$ as a function of $\omega$ could be expected for these values of $k$.

\section{References}

[1] I.M. de Schepper and E.G.D. Cohen, Phys. Rev. A22 (1980) 287; J. Stat. Phys. 27 (1982) 223.

[2] I.M. de Schepper, P. Verkerk, A.A. van Well and L.A. de Graaf, Phys. Rev. Lett. 50 (1983) 974; to be published.

[3] W.E. Alley and B.J. Alder, Phys. Rev. A27 (1983) 3158.

[4] I.M. de Schepper et al., Phys. Rev. A29 (1984) 1602.

[5] I.M. de Schepper, E.G.D. Cohen and M.J. Zuilhof, Phys. Lett. 101A (1984) 399.

[6] C.S. Wang Chang and G.E. Uhlenbeck, in: Studies in statistical mechanic, Vol. 5, eds. J. de Boer and G.E. Uhlenbeck (North-Holland, Amsterdam, 1970); J.D. Foch and G.W. Ford, in: Studies in statistical mechanics, Vol. 5, eds. J. de Boer and G.E. Uhlenbeck (North-Holland, Amsterdam, 1970).

[7] J.C. van Rijs, I.M. de Schepper and E.G.D. Cohen, to be published.

[8] J.-B. Suck, H. Rudin, H.-J. Güntherodt and H. Beck, Phys. Rev. Lett. 50 (1983) 49.

[9] B.M. Powell and G. Dolling, in: Rare gas solids, eds. M.L. Klein and J.A. Venables (Academic Press, New York, 1977).

[10] G.V. Chester, in: The helium liquids, eds. J.G.M. Armitage and I.E. Farquhar (Academic Press, New York, 1975). 